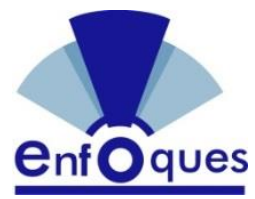

Enfoques. Revista de Investigación en Ciencias de la Administración http://doi.org/10.33996/revistaenfoques.v2i8.46 No. 8 | Volumen 2 | Octubre - Diciembre 2018 http://revistaenfoques.org

\title{
CONDICIONES INTEGRALES DE TRABAJO DE LOS RECOLECTORES DE DESECHOS SÓLIDOS DE UN MUNICIPIO EN VENEZUELA
} ISSN: $2016-8219$ pp. $242-264$

\section{COMPREHENSIVE WORKING CONDITIONS OF SOLID WASTE COLLECTORS OF A MUNICIPALITY IN VENEZUELA}

Glenys Gomes y Jaime Quintero

Artículo recibido julio 2018 | Arbitrado julio-agosto 2018 | Publicado 01 de octubre 2018

\section{Resumen}

El propósito del presente artículo consiste en describir las condiciones integrales de trabajo de recolectores de desechos sólidos de un municipio en Venezuela, con el fin de promover correctivos en el marco regulatorio venezolano y del trabajo decente, apoyado en la teoría Parsons, riesgo de Beck y 2 Factores de Herzberg. Fue una investigación de tipo cualitativa, descriptiva, basado en una estrategia, se apoyó en la lista de chequeo, observación y entrevista dirigida a informantes clave, clasificados en expertos, trabajadores, empresas, usuarios. Como resultados se tiene que la recolección de desechos, es un sistema que inicia desde el hogar con la producción de los desechos sólidos para luego proceder con la recolección y su traslado, es allí donde interactúan en un ambiente que impacta en los recolectores y se evidencia que en la actualidad no se está realizando de la forma adecuada, generando así un riesgo a la salud de los recolectores, en el municipio se está trabajando en la administración de la gestión ambiental, dirigiendo proyectos y programas a las comunidades para disminuir los efectos de contaminación, debido a que esta situación afecta directamente las condiciones de trabajo de los recolectores de desechos sólidos.

Palabras clave: Recolectores de desechos sólidos; condiciones integrales; marco regulatorio

\begin{abstract}
The purpose of this article is to describe the integral working conditions of solid waste collectors of a municipality in Venezuela, in order to promote corrections in the Venezuelan regulatory framework and decent work, supported by Parsons theory, Beck's risk and 2 Factors of Herzberg. It was a qualitative, descriptive research, based on a strategy, based on the checklist, observation and interview addressed to key informants, classified as experts, workers, companies, and users. As a result, it is necessary to collect waste, it is a system that starts from home with the production of solid waste and then proceeds with the collection and transfer, it is there that they interact in an environment that impacts the collectors and it is evident that at present it is not being carried out properly, thus generating a risk to the health of the collectors, in the municipality work is being carried out in the administration of environmental management, directing projects and programs to the communities to reduce the effects of pollution, because this situation directly affects the working conditions of solid waste collectors.
\end{abstract}

Key words: Solid waste collectors; integral conditions; regulatory framework

\author{
Glenys Gomes \\ glengomes@hotmail.es \\ Universidad de Carabobo, Venezuela \\ Licenciada en relaciones industriales \\ de la universidad de Carabobo. \\ Jaime Quintero \\ yojanderj20@hotmail.com \\ Universidad de Carabobo, Venezuela \\ Licenciado En Relaciones Industriales De \\ La Universidad De Carabobo. Cursos: \\ Fideicomiso, (INSBANCA) -PAS 20 (INCE)- \\ Organización, Dirección y Control de \\ Empresa (INCE).
}

(CC

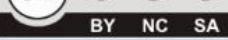

Revista Enfoques 


\section{INTRODUCCIÓN}

Las condiciones de trabajos son asumidas por Creus y Mangosio (2011) como factores que influyen en el hacer de las tareas que les son encargadas al trabajador, éstas son clasificadas en condiciones medioambientales; condiciones físicas en las que se realiza el trabajo; condiciones organizativas que rigen en la empresa en la cual se labora. Además, las condiciones integrales de trabajo pueden repercutir en la salud del trabajador, como en el proceso de trabajo relacionado con las condiciones de salud e higiene en el trabajo, lo cual deteriora la calidad de vida en el aspecto laboral. Con esto se puede determinar que la condiciones de trabajos están relacionadas con dimensiones de empleo, salario, seguridad social, salud laboral y su relación con lo eco social.

También es importante señalar que las condiciones de trabajo en términos de empleo, compensaciones y condiciones físico ambientales donde se ejecuta el trabajo están relacionadas con la concepción de trabajo decente que sostiene la OIT como instancia que fija los criterios de ejecución de prácticas destinadas a mejorar las condiciones de trabajo. Con trabajo decente se activan todos los mecanismos que conducen al mejoramiento permanente $\mathrm{y}$ sustentable de condiciones de trabajo dignas realizadas con protección a los derechos humano implícitamente ligados al mundo del trabajo.

Chiavenato (1989: 424).dice que las condiciones de trabajo son "las condiciones generales y especificas bajo las cuales se realiza la ejecución de las tareas", el resalta que existen "tres factores importantes los cuales son: iluminación, el ruido, las condiciones atmosféricas

Para Azuaje (2000) las condiciones de trabajo siempre han sido objeto de numerosas regulaciones, en defensa o resguardo de la salud del trabajador. Además, la Constitución de la República Bolivariana de Venezuela en el artículo 87 establece los mecanismos regulatorios de las condiciones materiales, morales e intelectuales de los trabajadores y establece los componentes por medio de los cuales se rigen las relaciones laborales. Los organismos internacionales tales como la Organización Internacional del Trabajo (OIT: 2000) tal como indicamos han implementado el concepto de trabajo decente.

Por ello, el presente artículo destaca el interés en el estudio de las condiciones integrales de trabajo de los recolectores de desechos sólidos del municipio Naguanagua estado Carabobo, Venezuela, que en la actualidad realizan esta labor sin las condiciones de trabajo mínimas, creando un sinfín de enfermedades propagadas en los trabajadores, generando como consecuencias que estas relaciones laborales no se apeguen a los establecido en la ley.

Es importante mencionar que en la Ley Orgánica del Trabajo los Trabajadores y las Trabajadoras en Venezuela en su artículo 156 se establece que:

El trabajo se llevara a cabo en
condiciones mínimas dignas y
seguras, que permitan a los
trabajadores y trabajadoras el
desarrollo de sus potencialidades,
capacidad creativa y pleno respeto
de sus derechos humanos. En su
numeral D) el ambiente saludable
de trabajo y E) la protección a la
vida, la salud y la seguridad
laboral.

Por otro lado, en La Ley Orgánica de Prevención, Condiciones y Medio Ambiente de trabajo en su artículo 59 norman las condiciones de trabajo dignos, por medio de las cuales deberán prestar los servicio 
patrono- trabajador y en el cual este deberá realizar la labor de acuerdo con los establecido en el artículo, es por ello que el artículo 11 del reglamento de la Ley Orgánica de Prevención, Condiciones y Medio Ambiente de trabajo dice "se entiende por condiciones de trabajo las condiciones generales y especiales bajo las cuales se realiza la ejecución de las tareas".

Las personas que prestan su servicio en los camiones recolectores de desechos sólidos, están sometidos a condiciones adversas a lo establecido en las normas nacionales y los tratados internacionales. En la actualidad los desechos son trasladados al vertedero que se encuentra ubicado en el municipio Libertador del estado Carabobo, este tiene alrededor de 40 años. Él denominado "Bote La Guasima" posee 57 hectáreas es el responsable de recibir al menos un aproximado de dos mil toneladas diarias de desechos sólidos, esto genera como consecuencia un ambiente contaminante para los trabajadores de los camiones recolectores de desechos, quienes al realizar esta labor sin los implementos de trabajo adecuados ponen en situación de riesgo la salud de los recolectores de desechos sólidos.

Esta situación genera inquietud porque afecta la vida de los trabajadores su desarrollo personal y se trasgrede la establecido la legislación venezolana. EI estudio condiciones integrales de trabajo de los recolectores de desechos sólidos del municipio Naguanagua, permitió dar respuestas.

Debido a la inquietud, con relación a las condiciones de trabajos, la finalidad que encontró este estudio fue describir las condiciones integrales de trabajo de los recolectores de desechos sólidos del municipio Naguanagua Edo. Carabobo, con el fin de promover correctivos a la luz del marco regulatorio venezolano y del trabajo decente. Para ello se analizó el entorno legal que ajusta las condiciones de trabajo de los recolectores de desechos sólidos. Como además, la evaluación de las condiciones socio-laborales en las que realizan el trabajo. Por último, busco la representación social de los trabajadores y los actores sobre el trabajo de recolección de desechos sólidos.

La investigación construyo un compendio de información que puede ayudar a las instituciones encargadas del funcionamiento de las condiciones integrales de trabajo de los recolectores de desechos sólidos quienes a su vez, deben velar por el cumplimiento y la aplicación de la normativa legal. Si bien es cierto que la Legislación Venezolana ha contemplado disposiciones que proporcionan las condiciones necesarias para el funcionamiento adecuado de las instituciones que emplean a estos trabajadores para brindar el servicio de recolección de desechos sólidos, es evidente que en la actualidad se encuentran desprotegidos aun y cuando el marco regulatorio establece sanciones como consecuencia del no cumplimiento de estas normas.

También, se desea destacar que las organizaciones que emplean a estos trabajadores generalmente son del estado quien a la vez es el órgano rector de estas normativas, por tal motivo se pone en evidencia que las practicas realizadas por estas empresas no son las adecuadas, colocando en situación de riesgo a los trabajadores que se dedican a la recolección de desechos sólidos. Esta investigación quiere dar una visión distinta del mundo del trabajo en el oficio de recolección de desechos sólidos.

Es importante destacar que la situación del vertedero es crítica y se encuentra de situación de riesgo ya que posee niveles de 
contaminación altos, que generan condiciones adversas a los trabajadores, la investigación pretende brindar información de las condiciones de trabajo a las que estos trabajadores están expuestos.

La superficie que comprende el relleno sanitario está completamente colapsada, se encuentran residuos de todo tipo y constantemente se producen quemas que emanan gases contaminantes produciendo un impacto ambiental negativo que afecta a los trabajadores que allí laboran, sin embargo la mayor complicación está en cuanto a las condiciones bajo las cuales realizan esta labor, es evidente que estos trabajadores no tiene estabilidad laboral, pocos cuentan con contrataciones que permitan normar o dar figura legal a su condición de trabajo. La investigación pretender brindar recomendaciones que procuren mejorar tales condiciones, y con ellos humanizar al recolector de desechos sólidos.

\section{MÉTODO}

El estudio se desarrolló bajo el paradigma cualitativo, de tipo descriptiva y se apoya en referencia documental siguiendo la línea de investigación de relaciones de trabajo. En la investigación que se desarrolló se estudiaron las características importantes del fenómeno objeto de estudio, relacionadas con las condiciones integrales de trabajo de los recolectores de desechos sólidos en el municipio Naguanagua en Venezuela. Se utilizaron como técnica e instrumentos de recolección de datos, la entrevista, guía de observación, lista de chequeo y la revisión documental, con el fin de recabar información. Se trabajó con bajo el sistema de grupos focales con los trabajadores de desechos sólidos.

\section{RESULTADOS Y DISCUSIÓN}

A continuación se desglosan los resultados arrojados en Lo que fue el estudio relacionado con la seguridad integral de los trabajadores de desechos sólidos del municipio Naguanagua en el estado Carabobo, Venezuela como consecuencia de haber administrado los elementos técnicos e instrumentales propuestos. Para ello, se recurrió a informantes claves quienes con su amplio conocimiento brindaron la información necesaria para contractar la realidad a la luz de las regulaciones laborales.

A los fines de la investigación se destaca, que tal como se establece en la teoría de sistemas, el proceso de recolección de desecho sólido, se inicia desde la clasificación en casa de los desechos, hasta el traslado de los mismos al relleno sanitario ubicado en el municipio Libertador del estado Carabobo conocido como vertedero la Gusima. Es aquí donde se procede a clasificar todos los desechos provenientes de los municipios del estado. Estos procesos se apoyan en la gestión ambiental. Mediante el desarrollo d se procedió con el análisis y la interpretación de los datos suministrados por los informantes a través de las técnicas e instrumentos utilizados en la investigación, tales como la entrevista, observación directa $\mathrm{y}$ lista de chequeo.

Es importante destacar que los instrumentos aplicados fueron validados por las instituciones públicas y fuentes tomadas de la jurisprudencia venezolana, en el caso de la lista de chequeo de inspección laboral es emitida por la inspectoría del trabajo y es válida para inspecciones de seguridad social, laboral y de condiciones de seguridad basada en las normativas legal vigente $y$ resoluciones en el área laboral. 
Con respeto al test de dependencia laboral, está establecido por la OIT a través de fuentes públicas para comprobar los procesos de tercerización laboral apoyados en lo establecido en la LOTTT. A continuación en figura 1 y cuadro 1 que detalla de qué manera se le dieron respuestas a los objetivos planteados en el desarrollo del estudio.

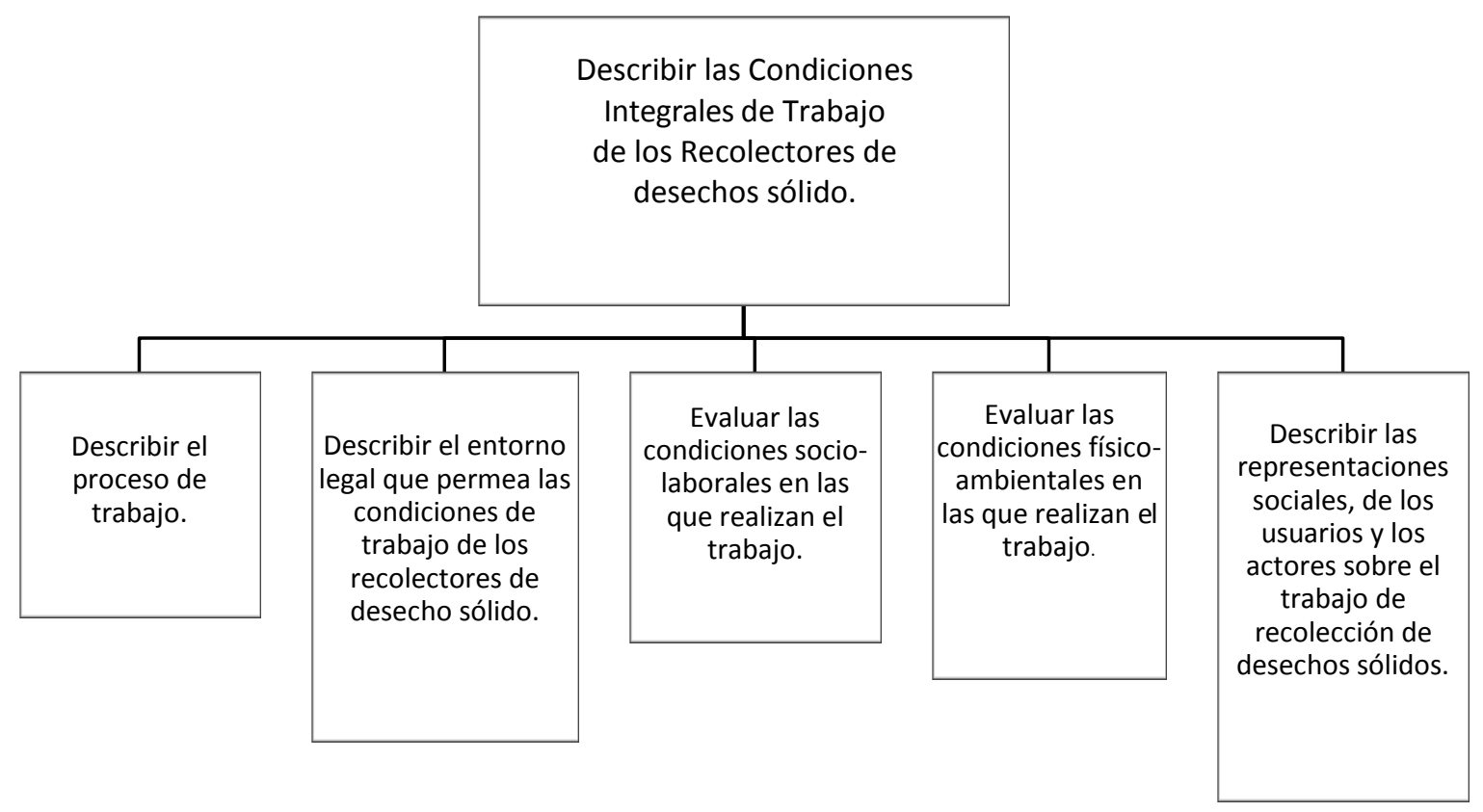

Figura 1. Esquemas de los objetivos 
Cuadro 1. Categorías del estudio sobre las condiciones integrales

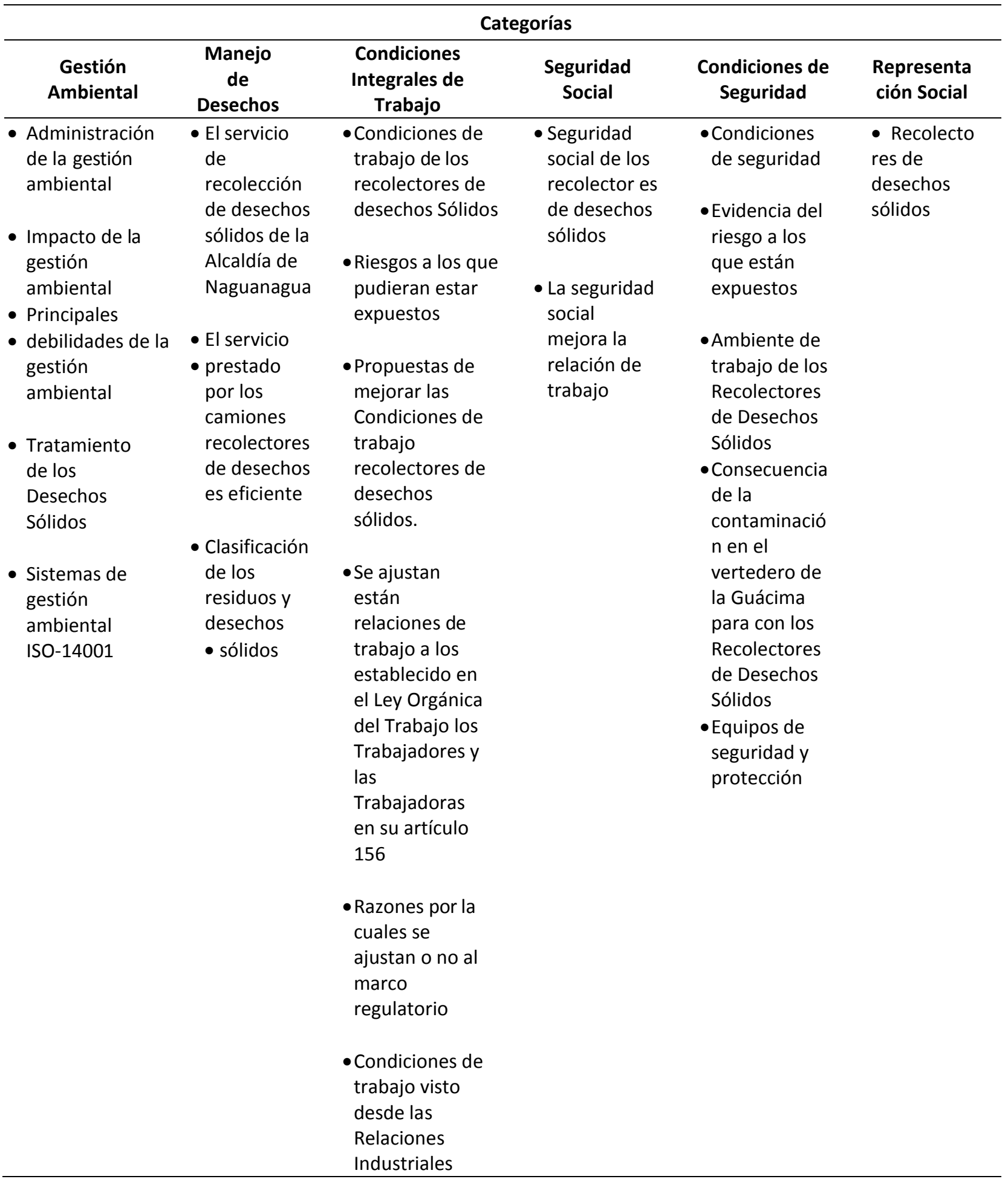


Los referentes teóricos utilizados en la investigación para dar respuestas a los objetivos planteados fueron la teoría de sistema que surge por la necesidad de abordar científicamente la comprensión de los sistemas concretos que conforman una realidad, generalmente complejas y únicas, resultantes de una historia en particular. En cuanto al proceso de la recolección de desechos sólidos parte desde la recolección hasta el traslado, al ambiente donde será clasificado y procesado por los actores involucrados en la actividad.

Según Parsons (1951) citado por Holm y Martin (2005) plantea que "un sistema social consiste en una pluralidad de actores individuales que interactúan entre sí en una situación que tiene, al menos, un espacio físico o medio ambiente" (p. 145), estas situaciones pueden repercutir en la acciones de los trabajadores. Es por ello, que las ciencias sociales se fundamentan en marcos de análisis que permiten establecer comparaciones a través del estudio de variables que afectan el desarrollo de los actores.

A través de la teoría de los sistema se observa el desarrollo de los actores y los efectos del ambiente en sus acciones y decisiones es importante destacar que al respecto Luhmann ( 1986:74) citado por Holm, Martin (2005) dice al respeto que "la teoría de la diferenciación funcional de sistemas es un instrumento explicativo de gran alcance, elegancia y económico para aspecto positivos y negativos de la sociedad moderna" las sociedades modernas en cuanto al procesos del manejo y recolección de desechos estableció nuevos estándares que si bien deberían accionarse la realidad es otra.

Es por ello que se utilizó la teoría del riesgo que se sustenta a través de la sociedad del riesgo es que una nueva forma social que surge como consecuencia de la modernización de la sociedad industrial.

Es por ello que según Albarracín (2002):

Beck categorizo el riesgo y estableció mediante la misma que la mayor parte de los desafíos que enfrentan los seres humanos en su vida social ya no provienen de la naturaleza, sino de las consecuencias de las propias acciones humanas. Las enfermedades, las maneras de alimentarse, de reproducirnos como especie, todo aquello que genéricamente era envuelto bajo el rubro intercambio con la naturaleza ha cambiado drásticamente de signo. Entre sus consecuencias está el paso de una lucha social por la apropiación del excedente a demandas por el control social del riesgo.

En la actualidad estos altos niveles de desechos se han transformado en contaminación ambiental. Sin embargo, el proceso de recolección de basura es parte de un sistema en el cual interactúan una serie de paso en las cuales el trabajador, se ve expuesto, colocando en riesgo no solo su salud, sino también su desarrollo psicosocial.

El análisis de los hallazgos, se elaboró a partir de las siguientes categorías fundadas en los análisis teóricos y proposiciones realizadas en el área, con el fin de establecer análisis comparativos de lo afirmado sólidos a la luz de nuestros referentes.

A los efectos del análisis de las categorías se presentaran de acuerdo al siguiente esquema.

- La gestión Ambiental desde la perspectiva de los actores laborales y nuestro especialista referencial.

- De la ordenanza municipal del servicio de los de Aseo Urbano a los hechos: El problema del manejo de los Desechos Sólidos. 
- Trabajo decente, calidad de vida laboral y condiciones Integrales de trabajo.

- Protección al Trabajo de Administración de Desechos Sólidos: La seguridad social en evidencia.

- El trabajo de la recolección de basura y las condiciones de seguridad.

- Valoración de la Gestión Ambiental desde la perspectiva de los Usuarios.

\section{La gestión ambiental desde la perspectiva de los actores laborales y nuestro experto referencial}

La gestión ambiental está relacionada con la investigación, ya que es el ambiente de trabajo de los recolectores de desechos sólidos y está directamente ligada sobre estos trabajadores, al respecto Bolca (1994) comenta que la gestión ambiental, es un "Conjunto de acciones que permitan lograr la máxima racionalidad en el proceso de toma de decisión relativa a la conservación, defensa, protección y mejora del ambiente, mediante una coordinada información interdisciplinaria y la participación ciudadana". es un proceso que se deriva de acciones comunes entre los administradores y la sociedad tal efecto está relacionado con lo establecido por breck en la teoría del riesgo, en cuanto al consumo excesivo y generación de los desechos sólidos, y la probabilidad de estar frente a riesgos como consecuencias de la acciones de la sociedad.

Es por ello, que la administración de los desechos sólidos se establece, mediante un proceso que permite a las instituciones procurar mecanismo de control y programas dirigidos al manejo efectivo de los desechos sólidos, apoyados en el reciclaje y la clasificación de los desechos, con el objetivo de tener sociedades más limpias y conservar el ambiente.
Expertos: Para el Informante I, la principal debilidad que se evidencia, es que no se aplica una buena administración de la gestión ambiental. Es importante destacar que la administración procura establecer medidas de control que permitan disminuir el efecto de la contaminación en el ambiente, a través de los programas dirigidos a las comunidades y diferentes alcaldías, el Estado debe establecer mecanismo para el control de la gestión ambiental con miras a promover una cultura de reciclaje. Hace referencia en cuanto a lo importante de contar con personas expertas en el área para tener una excelente administración, se infiere de una buena administración y control de la gestión, se generarían programas para el aprovechamiento de los desechos. Informante II, explico que existen proyectos establecidos por la alcaldía y esto repercute en el ambiente, de trabajo de los recolectores.

La importancia de controlar el ambiente es que esto incide directamente en el medio ambiente de trabajo de los recolectores y tienden a ser los factores que más afectan su desarrollo laboral y persona. Para Prigogine y Stengers, (1998) la gestión ambiental "plantea un problema conceptual que surge del cambio del paradigma determinista, basado en la simplicidad, uniformidad, independencia, estabilidad, control; por el paradigma de la incertidumbre, más complejo que se caracteriza por la complejidad, diversidad, interdependencia, dinamismo y riesgo". Esto como efecto de la globalización y el consumismo que plantea Beck en su teoría del riesgo, referente teórico de la investigación, ya que cada riesgo a los que están expuestos genera unas consecuencias que afectan en el desarrollo personal y de trabajo de los recolectores de los desechos sólidos. 
Trabajadores de las empresas contratistas: sobre el tema tienen poco conocimiento, desconocen si existen programas o si esto repercute en sus labores ni de las consecuencias del mismo en su labor.

Trabajadores por contrato de servicio: no poseen información acerca del tema. Se evidencia a través de la información brindada por los trabajadores que no poseen conocimiento de la gestión ambiental y las implicaciones que generan en el desarrollo de su trabajo. Sin embargo, las consecuencias de su impacto y posibles mejoras repercuten en el ambiente físico donde realiza la labor además de los efectos negativos evidente de la contaminación en los vertederos lugar donde culmina el proceso de trabajo, una vez recolectado los desechos, es trasladado para su tratamiento por parte de otra entidad de trabajo. Tales efectos están generando un gran impacto en su desarrollo personal y laboral según Munn, (1975) la "Actividad dirigida a identificar y predecir el impacto sobre la salud y el bienestar humanos, de propuestas legislativas, políticas, programas y procedimientos operacionales, y para interpretar y comunicar información sobre los impactos". Es por medio de actividades de control que se pueden evaluar las consecuencias en las condiciones de trabajo de los recolectores de desechos sólido. Es importante recordar que la Lopcymat establece que los trabajadores tienen derecho a un ambiente digno de trabajo.

Conclusiones: Con la información brindada por los informantes claves podemos concluir que la gestión ambiental en el estado Carabobo no se está llevando de la manera más adecuada poniendo en riesgo la salud de los recolectores de desechos sólidos así como la de sus habitantes. En el municipio Naguanagua se está trabajando en una gestión ambiental, dirigiendo proyectos y programas a las comunidades que permitan disminuir los efectos de la contaminación en el medio ambiente, esto incide directamente sobre las condiciones de trabajo de los recolectores de desechos sólidos mejorando su ambiente de trabajo y disminuyendo los riesgos a los que se expone.

De la ordenanza municipal del servicio de los de aseo urbano a los hechos: el problema del manejo de los desechos sólidos

El manejo de los desechos sólidos permite establecer tratamiento que van en pro de la conservación y la clasificación de los desechos y la incidencia en el ambiente de trabajo de los recolectores, es a través de este indicador que se desea conocer los efectos de la gestión ambiental y el manejo de desechos sólidos en las condiciones físico ambientales de los recolectores de desechos sólido.

Los residuos sólidos según Ruiz (2004) los desechos "son los restos de actividades humanas, considerados por sus generadores como inútiles, indeseables o desechables, pero que pueden tener utilidad para otras personas. La alcaldía de Naguanagua establece en su ordenanza Nro. 95 con respeto al aseo urbano, el proceso por medio del cual se establece este tratamiento por medio de cual se realiza la manipulación de estos desechos. Al respecto Busto (2008) define que, "En el caso de desechos sólidos municipales se aplican términos más específicos a los desechos de alimentos putrescibles (biodegradables) llamados basura, y a los desechos sólidos no putrescibles, los cuales se designan simplemente como desechos" y a través de esta afirmación que en manejo de los desechos debe hacer a través de una clasificación. 
Expertos: lo expuesto por el informante I al respecto del manejo de los desechos sólidos, es que la sociedad no se instruye con respecto al tema del reciclaje y la reutilización de objetos. Su visión está orientada a la importancia de la clasificación y la cultura de reciclaje al momento de hacer la separación de los desechos se debería aplicar planes para aprovechar al máximo los desechos, estos se apoyan en la gestión ambiental según Heer y Hagert, (1977) "Consiste en establecer valores cuantitativos para parámetros seleccionados que indiquen la calidad del ambiente, antes, durante y después de la acción", sin embargo El Informante II, asegura que en la actualidad se está implementando un programa piloto para dar respuesta al tema y las consecuencias para mejoras y aprovechar los desechos sólidos, el plan va orientado a la clasificación, según los mecanismo de las grandes organizaciones que recurren al reciclaje y los efectos que pueden generar en la sociedad.

Trabajadores de la Empresa Contratistas: Deben cumplir con unos requisitos previos tales como, los desechos sólidos estén bien embolsados y dispuestos en depósitos cerrados, si es centro comercial deben estar refrigerados para que el proceso de descomposición sea más lento. No trasladan los desechos vegetales como el carbón. Aseguran que solo trasladan los que estén en bolsas. Esto atreves de un criterio establecido por la contratista en cuando a las condiciones de traslado de los desechos.

Trabajadores por contrato de Servicio: existe un criterio propio y en principio hacen énfasis en el traslado de los desechos que estén en bolsas cerradas. Es por ello, que los recolectores de manera informal hacen una selección de los desechos, que trasladan para impactar lo menos posible sus herramienta de trabajo, entre alguna que mencionaron fue el camión utilizado para el traslado, tal como se lo afirma Parsons (1951) un sistema interactúa entre los actores y su individualidad, el espacio físico o medio ambiente, es esta integración lo que detalla el proceso de la recolección de desecho que inicia en la recolección hasta el punto de llegada del desecho y la interacción que existe entre la sociedad y la producción de los desechos. $Y$ incluso el nivel de consumo excesivo ha generado un incremento de desechos que tiende a impactar aún más el proceso de la recolección y el manejo de forma ineficiente de la clasificación de los desechos y el proceso de reciclaje en el municipio. Durante la investigación se conoció que en la actualidad la cuidad tiene un programa de clasificación de desechos una vez realizada la recolección y estos apoyados en las alcaldías sin embargo no ha sido efectivo en todas las comunidades.

Conclusiones: En el municipio Naguanagua, no se realiza manejo de desechos sólidos por parte de los recolectores, ya que estos se hacen en los hogares de los usuarios y estos trabajadores solo trasladan los desechos sólidos desde los sitios de almacenamiento locales a los camiones recolectores para luego ser llevados al vertedero la Guasima. Los expertos hicieron énfasis en la necesidad de educar en el tema del reciclaje y el manejo que se hacen de los desechos, logrando que el proceso de recolección de desechos sólidos sea más eficiente sin exponer a los recolectores a condiciones de trabajo insalubres. (Se recomienda ver anexo C fotografías). 
Trabajo decente, calidad de vida laboral y condiciones integrales de trabajo

Al referirse a condiciones integrales de trabajo se hace énfasis en lo establecido en el artículo 156 de la Ley Orgánica del Trabajo los Trabajadores y Trabajadoras (LOTTT) que establece las condiciones dignas y seguras de trabajo y como estas repercuten en el desempeño y desarrollo personal de los trabajadores. Para la Organización Internacional del Trabajo (OIT) es primordial establecer condiciones que permitan al trabajador su desarrollo, según Azocar y Díaz (2008), "La calidad de vida laboral puede también expresarse como el grado de satisfacción espiritual del hombre con el trabajo que realiza alcanzando su cima cuando el trabajo se convierte en su primera necesidad vital, de esta manera el empleado definiría el trabajo como la fuente esencial de la calidad de vida" es por ello que brindar condiciones de trabajo, no es solo referente a la estabilidad formal del trabajo, si no a la protección del trabajo a niveles de seguridad social, empleo, salario, seguridad laboral y los beneficios que la normativa legal establece.

Estas se fundamentan en las características de la definición del trabajo decente (2005) "sintetiza las aspiraciones de las personas durante su vida laboral. Significa la oportunidad de acceder a un empleo productivo que genere un ingreso justo, la seguridad en el lugar de trabajo y la protección social para las familias, mejores perspectivas de desarrollo personal e integración social".

Expertos: Informante I, establece que es evidente que no cuentan con condiciones dignas de trabajo, esto a consecuencia de que la sociedad no tiene cultura en cuanto al manejo de desechos sólidos y esto trae como consecuencia que, en muchas ocasiones a los trabajadores se les afecte su integridad física, además de las consecuencia que existen en el manejo de los desechos, por parte de la sociedad. Se sostiene que las condiciones de trabajo están constituidas por múltiples variables, el informante sustenta que las condiciones de seguridad personal de los recolectores de desechos sólidos son las más afectadas, y que el artículo 59 de la LOPCYMAT donde establece las condiciones y lineamientos mínimos para que un trabajador realice su labor en condiciones segura y efectiva no se cumple. En cuanto a este tema la informante II asegura que en la entidad laboral tienen respeto por su desarrollo físico y moral y las condiciones mínimas establecidas en su contrato de trabajo, cuentan con los equipos de seguridad necesarios para proteger su integridad personal, hace énfasis en el respeto para con su desarrollo personal brindado por la empresa.

Sin embargo, Neffa (2015) establece que las condiciones y medio ambiente de trabajo "están constituida por un conjunto de variables que a nivel de la sociedad son un conjunto". Tal conjunto incide en la realización de las labores; Es por ello, que si los recolectores cuentas con beneficios y contratos de trabajo destinados a proteger al trabajador desde la perspectivas de estabilidad laboral, los mismo se comprometerán a realizar su actividad de la manera más eficiente, sin embargo, Breck establece en su teoría del riesgo que, ocurre el riesgo si la sociedad procurar tener riesgo, los trabajadores esta en riesgos por decisión propia o por obligación y dependencia.

Trabajadores de la empresa contratista: aseguran que en este momento la infraestructura del punto de control no está apto pero a consecuencias de factores externos. Se sienten seguro gracias a que sus condiciones de trabajo están establecidas en 
el contrato, aseguran contar con lo establecido en las normativas legales vigentes. Además, cuentan con contrato escrito de trabajo, salario y bono de alimentación. De acuerdo a lo establecido en las normativas ellos cuentan con el beneficio de seguro social. Se infiere que la entidad laboral está cumpliendo con su responsabilidad ante sus trabajadores sin embargo es esta entidad es quien realiza la contratación de los camiones independiente, aseguran que es como apoyo por necesidad de camiones de traslado.

Trabajadores por contrato de servicio: A diferencia de los recolectores que prestan servicio en la entidad de trabajo, se logró evidenciar que estos recolectores no tienen relación laboral con la contratista si no un contrato de servicio. Además no cuentas con condiciones dignas de trabajo, sin contrato escrito, sin estabilidad laboral sus condiciones no se ajustan a lo establecido en las normativas legales. Generalmente son los que cubren las rutas con más inconvenientes y estados de contaminación que afectan de forma directa su salud. Esto impacta en su salud y no cuentan con la estabilidad que brinda el contrato escrito, ni jornada fija de trabajo en ocasiones su jornada inicia a las 4 am y culmina a las $7 \mathrm{pm}$ todo dependiendo de las rutas que realicen.

Conclusiones: los recolectores de desechos sólidos no cuentan con condiciones dignas de trabajo, ya que se vulnera las condiciones de seguridad de los recolectores y que los trabajadores que prestan un servicio a la empresa contratista no cuentan con una estabilidad laboral dejando en evidencia que existe una relación de trabajo de forma tercerizada, aunque los recolectores de desechos sólidos perteneciente a la empresa contratista se le respeta las condiciones mínimas de trabajo.

\section{Protección al trabajo de administración de desechos sólidos: la seguridad social en evidencia}

La Seguridad Social es un derecho para todos los trabajadores, al respeto Colombet (2013), "indica que el riesgo es todo aquel acontecimiento que puede ocurrirle a una persona, que no sea producto de su voluntad" (p. 91). Es por ello, que la seguridad social comprende todo lo relacionado con las condiciones de seguridad establecidas en la normativa, contar con la cobertura de riesgo que permitan al trabajador gozar de una pensión y de estabilidad ante un accidente o discapacidad por enfermedades.

Es importante destacar que existe mayor posibilidad de accidentes laborales, al no tener control en el medio ambiente de trabajo, esto eleva la probabilidad de padecer enfermedades ocupacionales $y$ accidentes, en el caso de los recolectores de desechos sólidos están expuestos a ambientes que no se pueden controlar directamente por las entidades de trabajo. Esta labor al transcurrir el tiempo puede generar enfermedades ocupacionales $y$ accidentes de trabajo, el sistema de seguridad social procura proteger al trabajador ante cualquiera de estas contingencias. Como se procede en la alcaldía en cuanto a la seguridad social de los trabajadores, aunque por lo apreciado parece que no cuentan con los requisitos mínimos de seguridad al realizar el trabajo, considera que el contar con un seguro o un HCM permitiría al trabajador, estar amparado y contar con un beneficio que debe ser un derecho al respeto La Ley del Seguro Social Artículo 2 (2012) establece "bajo la inspiración de la justicia social y la equidad, a la progresiva aplicación de los principios y normas de seguridad social a 
todos los habitantes del país. Están protegidos por el seguro social obligatorio", sin embargo la realidad de los trabajadores se infiere que es otra, en cuanto a los trabajadores de contrato de servicio. Informante II asegura que los trabajadores están amparados bajo el sistema de seguridad social, ya que ellos están inscritos y cuentan con lo establecido en la normativa. Estos trabajadores están bajo la protección del seguro ya que cuentan con un patrono que asegura estar solvente con la seguridad de sus trabajadores, para que los mismos pueden retirarse en tranquilidad al momento de llegar a su vejez, o retirarse por enfermedad $\mathrm{o}$ accidente laboral, la importancia de esta ley del seguro social radica en la protección a la salud del trabajadores.

Trabajadores de la Empresa Contratistas: Estos trabajadores cuentan y están amparados bajo el sistema de seguridad social. Para con ellos se cumplen con sus derechos, disponen de las prestaciones por enfermedad o accidente establecidos en la ley. Ante la probabilidad de una contingencia derivada del riesgo cuentan con la seguridad.

Trabajadores por contrato de Servicio: Son trabajadores a destajo y en ocasiones trabajan por día, no cuentan con una estabilidad laboral por lo tanto no poseen seguro social, sin embargo es importante destacar que en la declaración de los derechos humanos, en el derecho a la seguridad social establece:

Toda persona como miembro de la sociedad, tiene derecho a la seguridad social, y a obtener, mediante el esfuerzo nacional y de cooperación internacional, habida cuenta de la organización y los recursos de cada Estado, la satisfacción de los derechos económicos, sociales y culturales indispensables a su dignidad y al libre desarrollo de su personalidad.

Es por ello que se evidencio que está en fragante violación de sus derechos humanos al prestar el servicio de recolección sin contar con seguridad social y beneficio mínimo, y estar sin estabilidad laboral.

Conclusiones: para los investigadores, los recolectores de desechos sólidos tienen que estar amparados en el sistema seguridad social siendo este un derecho obligatorio en la legislación venezolana, ya que el trabajo que realizan es de alto riesgo, teniendo mayor probabilidad de accidentes laborales como de enfermedades ocupacionales, y en estos casos el sistema de seguridad social procura proteger al trabajador ante cualquiera de estas eventualidades.

\section{El trabajo de la recolección de basura y las condiciones de seguridad}

En las organizaciones es de vital importancia velar por las condiciones de seguridad de los trabajadores y estas sean adecuadas a la labor que realizan y apegadas al marco regulatorio en esta materia, es por ello que las condiciones de seguridad en el trabajo son un área interdisciplinaria estrechamente vinculada con la seguridad, la calidad de vida y la salud dentro del trabajo. Al respecto Fernández (2005), define que "La seguridad en el trabajo abarca el conjunto de medidas empleadas para prevenir accidentes y eliminar las condiciones inseguras del ambiente, así como para instruir o convencer a las personas acerca de la necesidad de implementar prácticas preventivas."

Es sumamente importante garantizar las condiciones de seguridad al trabajador, así como también es de vital influencia dirigir charlas a los mismos sobre sus condiciones y el uso adecuado de sus equipos de trabajo. 
Expertos: Para el Informante I las condiciones de seguridad, de los recolectores de desechos sólidos es mala, porque aunque la alcaldía lo quiera hacer bien. En Venezuela no se clasifican los desechos por ejemplo un químico que se utiliza para limpieza si se mezcla con otro producto que no es compatibles puede generar una mayor contaminación esto como consecuencia de la inadecuada manipulación de los desechos. Aunado a estos los riesgos a los que se exponen, empezando porque estos trabajadores van guindados en un camión es un riesgo ya que podría sufrir una caída de un nivel a otro, porque a veces algunos trabajadores van en la parte alta del camión, y si hay un accidente van hacer golpeados, riesgo de cortaduras porque aunque usen los guantes puede ser que manipulando las bolsas este un objeto cortante o punzo penetrante que lo puede causar una herida. Ellos están expuestos algún desecho orgánico ya con salmonella y por descomposición o la picadura de algún animal, porque en una bolsa de esas puede venir una culebra o un alacrán tienen machismos riesgos animales muertos o productos descompuestos. Para el informante II Los recolectores del aseo urbano, trabajan en las unidades que prestan el servicio de aseo y estas se encuentran en buenas condiciones. Al igual que el galpón ubicado en el Municipio Libertador, en Naguanagua estamos en proceso de construcción del área de operaciones. Sin embargo, el vertedero la Guasima que forma parte de sus condiciones de trabajo, no cumple con los requerimientos mínimos.

Las condiciones de seguridad de los recolectores de desechos sólidos no son las más óptimas para el desenvolvimiento físico y mental de su labor, trayendo consigo posibles consecuencias negativas para su salud lo cual no permite un desempeño optimo en su área de trabajo.

Al respecto Chiavenato (2007) plantea que la seguridad laboral, como un conjunto de medidas técnicas, educativas, médicas y psicológicas utilizadas para prevenir accidentes, sea con la eliminación de condiciones inseguras del ambiente, con la instrucción o convencimiento de las personas para que apliquen prácticas preventivas, lo cual es indispensable para un desempeño satisfactorio del trabajo. Además que las condiciones de seguridad permiten que el trabajo, se desarrolle de forma efectiva y cuente con los mecanismos de control que permitan salvaguardar su salud y seguridad personal.

Trabajadores de la empresa contratistas: sobre el tema comentaron que sus condiciones de seguridad no son las mejores, ya que en este momento está en construcción las instalaciones para los trabajadores donde se encuentra el lugar de transferencia de los desechos. Expresaron que utilizan los implementos de seguridad y que el riesgo más grande que tienen es el de caer del camión recolector de desechos (Riesgo Físico).

Trabajadores por contrato de servicio: Expresaron que no cuentan condiciones de seguridad y que al riesgo al que más se exponen es al de caer del camión recolector de desechos (Riesgo Físico), porque a la hora de una caída los gastos médicos corren por el trabajador que presta el servicio. Explicaron que no cuentan con los implementos de seguridad necesarios para la realización de la labor, y desconocen los otros riegos a los que están expuestos en sus áreas de trabajo.

Conclusiones: las condiciones de seguridad de los recolectores de desechos sólidos no son las más adecuadas ya que estos trabajadores están expuestos a una 
cantidad de riesgos laborales que derivan de prestar este servicio. Los recolectores de desechos están expuestos a Riesgos Fiscos, Biológicos y Disergonómicos que les puede ocasionar enfermedades ocupacionales o accidentes laborales en sus aéreas de trabajo. Aunado a esto los recolectores de desechos sólidos no cuentan con los implementos de seguridad necesarios para llevar a cabo su trabajo de forma satisfactoria, exponiendo al trabajador a los riesgos antes mencionados.

Estos trabajadores están expuestos a condiciones de seguridad que ponen en riesgo su salud, es por ello que las empresas que se dedican a la recolección de desechos sólidos deben hacer un esfuerzo para prevenir los riesgos laborales que derivan de estas prácticas, a través de planes de formación en seguridad y salud laboral así como el uso adecuado de sus implementos de seguridad se logre disminuir los riesgos a los que se exponen los recolectores de desechos sólidos.

\section{Valoración de la gestión ambiental desde la perspectiva de los usuarios}

La representación social se encontró vinculada con esta investigación ya que los usuarios se encuentran de manera directa o indirectamente ligada al proceso de trabajo de los recolectores de desechos sólidos.

Al respecto Jodelet, 2000, citado por Perera, 2005

Las representaciones sociales conciernen al conocimiento de sentido común que se pone a

\begin{abstract}
disposición en la experiencia cotidiana; son programas de percepción, construcciones con status de teoría ingenua, que sirven de guía para la acción e instrumento de lectura de la realidad; sistemas de significaciones que permiten interpretar el curso de los acontecimientos y las relaciones sociales; que expresan la relación que los individuos y los grupos mantienen con el mundo y los otros; que son forjadas en la interacción y el contacto con los discursos que circulan en el espacio público; que están inscritas en el lenguaje y en las prácticas; y que funcionan como un lenguaje en razón de su función simbólica y de los marcos que proporcionan para codificar $y$ categorizar lo compone el universo de la vida. (p. 47).
\end{abstract}

Es por ello que es necesario conocer cómo se percibe al trabajador que se dedica a la recolección de desechos sólidos por parte de los ciudadanos que reciben este servicio con el fin de que estos trabajadores se inserten en la sociedad, sin menos cabo de sus condiciones sociales

\section{Análisis del discurso}

A continuación se muestra el cuadro 2 , acerca de la Representación social El cual da cuenta de la información brindada por los informantes y se recurre a un comparativo categórico. Por medio del siguiente cuadro se pretende evaluar la valoración de la gestión ambiental desde la perspectiva de los usuarios. 


\section{Cuadro 2. Representación social}

\begin{tabular}{|c|c|c|c|}
\hline $\begin{array}{c}\text { Informantes claves } \\
\text { categorías }\end{array}$ & Informante I & Usuario I & Usuario II \\
\hline $\begin{array}{l}\text { Representaciones } \\
\text { sociales }\end{array}$ & $\begin{array}{l}\text { Para este trabajo son las } \\
\text { personas que van } \\
\text { guindando en los camiones, } \\
\text { esas personas deben recibir } \\
\text { una educación para que } \\
\text { ellos sepan y las } \\
\text { condiciones mejorarlas, } \\
\text { porque los podemos } \\
\text { mejorar a ellos pero si los } \\
\text { sitios donde están ellos } \\
\text { recogiendo están en malas } \\
\text { condiciones porque la } \\
\text { gente no tiene una cultura } \\
\text { y la basura la rompen la } \\
\text { tiran, primero que como } \\
\text { país damos una triste } \\
\text { imagen porque no } \\
\text { haycontenedores para } \\
\text { reguardar las bolsas } \\
\text { plástica o los recipientes } \\
\text { donde se resguarda el } \\
\text { desecho antes que llegue el } \\
\text { camión, entonces los } \\
\text { perros, los gatos todos } \\
\text { estas animales rompen las } \\
\text { bolsas el recolector que } \\
\text { más va hacer lo hace } \\
\text { inadecuado porque mucho } \\
\text { de las condiciones de ellos } \\
\text { son las propicias }\end{array}$ & $\begin{array}{l}\text { Generalmente cuando } \\
\text { están realizando la labor } \\
\text { no cuentan con las } \\
\text { condiciones de } \\
\text { seguridad mínimas } \\
\text { como el uso de guantes, } \\
\text { ellos tocan los desechos } \\
\text { son sus manos, y no } \\
\text { usan faja para cuidar su } \\
\text { espalda hay bolsas que } \\
\text { son muy pesadas y en } \\
\text { ocasiones la basura } \\
\text { tiene gusanos., no se } \\
\text { sabe si se lo entregan } \\
\text { que no les han los } \\
\text { entregado. }\end{array}$ & $\begin{array}{l}\text { Considero que hacen } \\
\text { un trabajo difícil al } \\
\text { tratar de recolectar } \\
\text { los desechos que } \\
\text { nosotros se echamos, } \\
\text { yo pienso que } \\
\text { deberían ser tratados } \\
\text { con más respeto y } \\
\text { consideración él hace } \\
\text { ese trabajo no los } \\
\text { hacen menos. } \\
\text { Considero que son } \\
\text { eficiente aun y } \\
\text { cuando no cuentan } \\
\text { con condiciones de } \\
\text { salud. }\end{array}$ \\
\hline
\end{tabular}

Expertos: Para el Informante I: los recolectores de desechos sólidos son las personas que van guindando en los camiones, estos trabajadores deben recibir una educación para que conozcan sus condiciones y mejorarlas. Teniendo en cuenta que podemos mejorarlos a ellos, pero si los sitios donde están ellos recogiendo los desechos están en malas condiciones porque la gente no tiene una cultura de reciclaje y la basura la rompen, la tiran a las calles quedando expuestas por muchos días, damos una triste imagen como país ya que no existen contenedores para resguardar las bolsas plásticas o los recipientes donde se depositan los desecho antes que llegue el camión. Trayendo como consecuencia que perros, gatos y demás animales rompan las bolsas y el trabajador que más va hacer, realizar la recolección de manera inadecuada porque las condiciones no son las propicias. Informante II, los recolectores de desechos 
sólidos es un personal necesario y técnicamente preparado para desarrollar la recolección de los residuos y desechos sólidos residenciales de manera apropiada y que se trabaja para que ellos tengan mejores condiciones tanto ambientales como sociales.

Es importante conocer como los usuarios ven a los recolectores de desechos sólidos en el ámbito social ya que esto repercute en su desarrollo laboral, personal $\mathrm{y}$ en la motivación que tienen estos trabajadores en la consecución del logro de los objetivos de su trabajo. Para Banchs (1990) concibe "la representación social una modalidad de pensamiento práctico que sintetiza la subjetividad social. Está orientada hacia la comunicación, la comprensión y el dominio de su entorno social". Tal como lo plantea Frederick Herzberg en su teoría de los 2 factores que se encuentra en el referente teórico en el referente teórico de la presente investigación.

Usuarios: sobre el tema comentan que los recolectores de desechos sólidos no cuentan con las condiciones de seguridad mínimas como el uso de guantes para la recolección, y que no saben si es porque la empresa no les da la dotación de equipos de seguridad o porque los trabajadores no los usan, argumentaron también que a estas personas hay que darles educación sobre el uso de los implementos de seguridad porque pueden contraer enfermedades que atente contra su salud y la de los ciudadanos a su alrededor ya que muchas personas colocan en las bolsas de basuras jeringas sin colocarlo de manera correcta en los recipientes. Opinaron además que la labor que realizan estos trabajadores es de vital importancia para la salubridad de la sociedad.

Conclusiones: para los usuarios, los recolectores de desechos sólidos son unos trabajadores que prestan un servicio de gran valor a la sociedad, ya que ayudan a mantener las calles y avenidas limpias y la salubridad en las comunidades, mostrando gran preocupación por los recolectores de desechos ya que estos deberían utilizar los equipos de seguridad necesarios para reguardar su integridad física y prevenir los riesgo a los que se expone, que las empresas pertenecientes los incentiven a tomar planes de formación sobre los riesgo a los que están expuestos y al uso adecuado de los implementos de seguridad.

A fines de la investigación se aplicó una lista de chequeo de inspección laboral emitida por la inspectoría del trabajo basada en la normativa legal vigente y resoluciones en el área laboral, para dar respuesta a los objetivos siguientes: Describir el entorno legal que permea las condiciones de trabajo de los recolectores de desecho sólido; Evaluar la condiciones socio- laborales en las que realizan el trabajo; y Evaluar las condiciones físico-ambientales en las que realizan el trabajo. Se recomienda ver anexo C captura de fotografías.

\section{El trabajo, los trabajadores y trabajadoras desde la inspección laboral}

Análisis de la Inspección Laboral. El siguiente análisis se aplicó bajo la estructura de la lista de chequeo, elaborada por la inspectoría del trabajo, estableciendo criterios legales y apoyados en la legislación nacional. 
Cuadro 3. Inspección laboral

\begin{tabular}{|c|c|c|}
\hline Referente & Empresa contratista & $\begin{array}{c}\text { Camiones de contrato } \\
\text { de servicio }\end{array}$ \\
\hline $\begin{array}{l}\text { - Artículos Nro. 59, 63, 64, } \\
\text { 73,106,117,118,119,120,121,122 } \\
\text {, 128,129, 130, 131, 132, 133, } \\
\text { 136, 137, 138, 139, 140, } 142 \\
\text { literal a) y b), 143,144,167,168, } \\
\text { 175,176, 178, 182,183, } \\
\text { 185,188,190, 191, 192, 203, Art. } \\
173 \text { y Disposición Transitoria } \\
\text { Tercera LOTTT } \\
\text { - Artículo } 78 \text { RLOT, Artículos } 28 \\
\text { y } 90 \text { CRBV. }\end{array}$ & 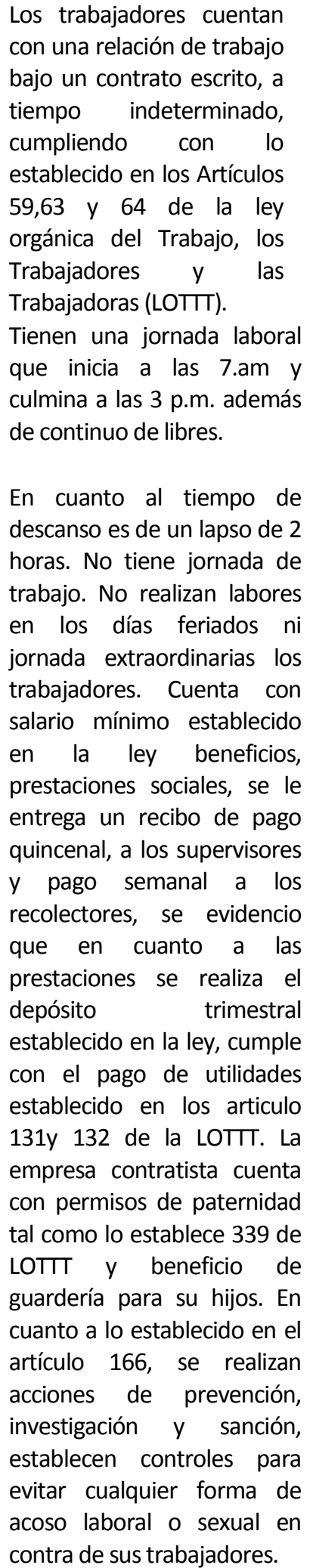 & 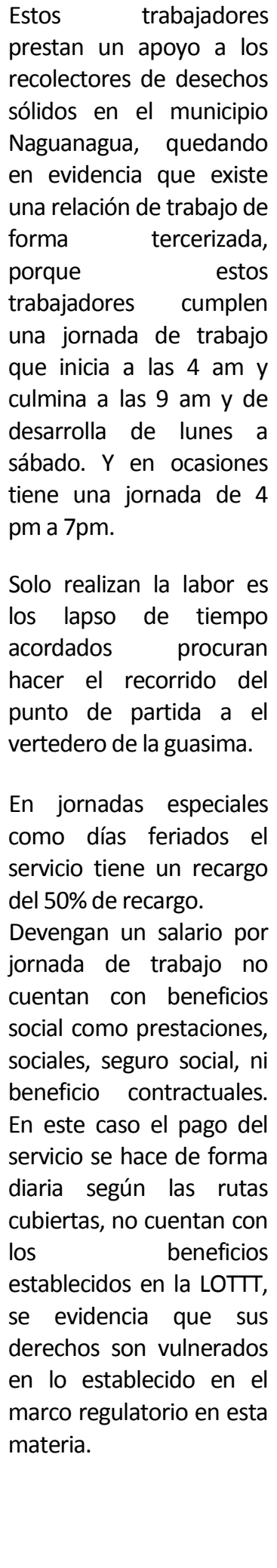 \\
\hline
\end{tabular}


Cuadro 4. Inspección de condiciones de seguridad

\begin{tabular}{l} 
Referente \\
\hline - Decreto Presidencial 2195 de \\
Fecha $17 / 08 / 83$ Gaceta Oficial \\
3270, de fecha 31/10/83 \\
- Convenio 120 OIT \\
- artículos $163,166319,32,305$, \\
27,289, 290, 291,292, 73, 190, \\
44, \\
156,158,43 de la LOTTT \\
- Artículos 10 y 15 de Ley del \\
INCESS
\end{tabular}

- Artículos 28 de la LPCD

- Artículos 55, 59, 63, 102, 103, 99 y 109 RPRGLSS

- Artículos 30 y 31 LRPVH

- Artículos 2 y 4 DRVFLAT 6 DRVFLAT (Parágrafo Único)

- Artículos 15, 16, 21, 24, 17, 18 RLAT

- Artículos 23, 80, 83 y 84 RPLOPCYMAT

- Artículos 6; 15; 22; 39; 40 numeral. 5; 41; 44; 46; 49 numeral $2 ; 53$ numeral $4,10,13$ y $14 ; 56$ numeral $3,4,7,8$ y 11 ; 58, 62 Numeral. 3, 73, 59 numeral 2 y $7 ; 60,62,65$ LOPCYMAT

- Artículos 12; 14; 15; 18; $22 ; 23$; 58; 78; 84; 85; 87; 93; 94; 95; 96 ; 97 ;100; 101; 103; 122; 128; $129 ; 133 ; 134 ; 137 ; 140 ; 141$ 144; 146; 147; 196; 311; 334; 494; 495; 496; 769; 770; 771; 772; 773; 776; 777; 778; 793; 862; 864; 866 del RCHST
Camiones de contrato de servicio

De la misma este camión tampoco cuenta con extintores como medida de seguridad para los trabajadores, estos se encuentran expuestos a caídas por aberturas del piso y caídas desde la parte más alta de camión, los mismo no cuentan con suministro de agua potable ni de comedor es por ello que lo realizan en el camión rodeado de todos estos desechos, no cuentan sanitarios para cubrir sus necesidades, y es importante destacar que estos trabajadores no cuentan con implementos de seguridad necesarios para llevar a cabo su trabajo exponiéndose a la cantidad de riesgos que derivan del proceso de descomposición de estos desechos. 
Cuadro 5. Inspección de seguridad social

\begin{tabular}{|c|c|c|}
\hline Referente & Empresa contratista & Camiones de contrato de servicio \\
\hline 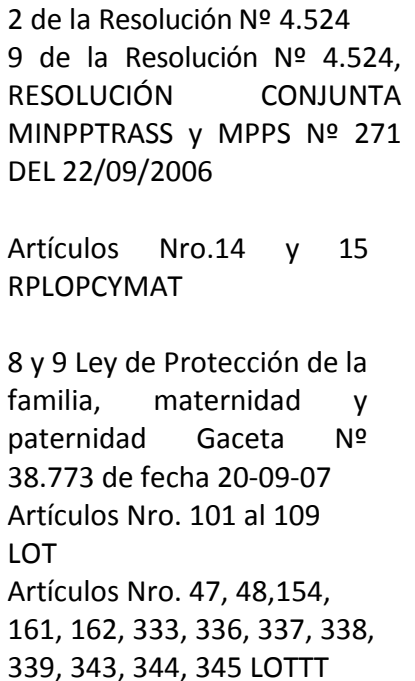 & $\begin{array}{l}\text { Cuenta con planes de formación y se } \\
\text { apoyan con la gestión ambiental de } \\
\text { los programas de cultura de la alcaldía } \\
\text { de Naguanagua, sin embargo, cuentan } \\
\text { con los planes de formación } \\
\text { establecido por INCES están inscritos } \\
\text { en el seguro social y cuentan con } \\
\text { protección del sistema de seguridad } \\
\text { social. En el caso de los delegados de } \\
\text { prevención y comité de seguridad y } \\
\text { salud laboral. Se demostró que si tiene } \\
\text { representantes. }\end{array}$ & $\begin{array}{l}\text { No cuentan con seguridad social y } \\
\text { tiene conocimiento de la los } \\
\text { beneficios y derechos establecidos } \\
\text { en la normativa legal. Desconocen } \\
\text { los planes de formación. Es evidente } \\
\text { la falta de estabilidad laboral para } \\
\text { estos trabajadores no dependientes } \\
\text { y en estado de tercerización. }\end{array}$ \\
\hline
\end{tabular}

Conclusiones: al finalizar la inspección en materia de empleo queda en evidencia la existencia de tercerización con los trabajadores que le prestan un servicio a la empresa contratista por demostrarse que existe una relación de trabajo, a estos trabajadores se les cancela diario por el servicio prestado, sin gozar de beneficios laborales como los que poseen los recolectores que trabajan para la empresa contratista, estos trabajadores si gozan de una relación de trabajo estable con beneficios contractuales mínimo establecido en la normativa legal. En la inspección de seguridad se demostró que los equipos para la recolección de desechos sólidos (camiones recolectores) no cuentan con las condiciones de seguridad necesarias para el desenvolvimiento pleno de los trabajadores, al igual que no cuentan con los implementos de seguridad para protegerse ante los riesgos que se exponen en su área de trabajo, no cuentan con suministro de agua potable durante el recorrido, así como tampoco cuentan con baños ni sala de descanso en el denominado punto de control, aunque cabe destacar que se encontraba en construcción al momento de la inspección. Al culminar la inspección de seguridad social se evidencio que los trabajadores que prestan un servicio a la empresa contratista no cuentan con seguridad social no tienen conocimiento sobre los planes de formación, los trabajadores perteneciente a la empresa contratista están inscrito en el sistema seguro social, cuentan con planes de formación y se demostró que cuentan con representantes en el comité de seguridad y salud laboral de la empresa.

\section{CONCLUSIONES}

De acuerdo a lo establecido por Parsons, sobre la teoría del sistema se infiere que el proceso de la recolección de desechos, es un sistema que inicia en el hogar con la producción de los desechos sólidos para luego proceder con la recolección y su traslado, es allí donde interactúan en un ambiente que impacta en los recolectores y 
se evidencia que en la actualidad no se está realizando de la forma adecuada, generando así un riesgo a la salud de los recolectores de desechos sólidos así como la de sus habitantes.

Es importante destacar que si el vertedero de desechos sólidos denominado la Guasima ubicado en el municipio Libertador, en el estado Carabobo se encuentra cerrado, se interrumpe el ciclo del proceso y los recolectores deben esperar para poder hacer el depósito de los desechos, ocasionando que el proceso de descomposición de los mismos se acelere e impacte en la salud de los trabajadores.

En el municipio Naguanagua se está trabajando en una administración de la gestión ambiental, dirigiendo proyectos y programas a las comunidades que permitan disminuir los efectos de la contaminación en el medio ambiente, esto incide directamente sobre las condiciones de trabajo de los recolectores de desechos sólidos, sin embargo, no se realiza manejo de desechos sólidos por parte de los recolectores, ya que estos se hacen en los hogares de los usuarios y estos trabajadores solo trasladan los desechos sólidos desde los sitios de almacenamiento locales a los camiones recolectores para luego ser llevados al vertedero la Guasima.

Es por ello, que se considera que es necesario la educación en cuanto al tema del reciclaje y el manejo de los desechos. Con relación a las condiciones integrales de trabajo, se evidencia que estas categorías anteriores repercuten de forma directa en las condiciones y el medio ambiente de trabajo de los recolectores de desechos sólidos. durante la investigación se evidenció que los recolectores no cuentan con condiciones dignas de trabajo, ya que se vulnera las condiciones de seguridad de los recolectores y que los trabajadores que prestan un servicio independiente no cuentan con una estabilidad laboral dejando en evidencia que existe una relación de trabajo de forma tercerizada, sus condiciones de seguridad son mermadas al estar expuestos a espacios con niveles altos de contaminación y factores que inciden de forma directa en sus salud y desarrollo personal, es importante destacar que solo los trabajadores de las empresas contratistas están amparados en el sistema de seguridad social siendo este un derecho obligatorio en la legislación venezolana, ya que el trabajo que realizan es de alto riesgo, teniendo mayor probabilidad de accidentes laborales como de enfermedades ocupacionales, y en estos casos el sistema de seguridad social procura proteger al trabajador ante cualquiera de estas eventualidades.

Sin embargo, para los usuarios, los recolectores de desechos sólidos son unos trabajadores que prestan un servicio de gran valor a la sociedad, ya que ayudan a mantener las calles y avenidas limpias y la salubridad en las comunidades, mostrando gran preocupación por las condiciones integrales de trabajo los recolectores de desechos ya que estos deberían utilizar los equipos de seguridad necesarios para reguardar su integridad física y prevenir los riesgo.

Materia de empleo quedo en evidencia la existencia de tercerización con los trabajadores que le prestan un servicio a la empresa contratista al demostrarse que existe una relación de trabajo, a estos trabajadores se les cancela diario por el servicio prestado, sin gozar de beneficios laborales. Esta información se apoya en el test de dependencia laboral aplicado durante la observación e investigación, quedando en evidencia la realidad a la que estos trabajadores están expuestos. 
En la inspección de seguridad se demostró que los equipos para la recolección de desechos sólidos (camiones recolectores) no cuentan con las condiciones de seguridad necesarias para el desenvolvimiento pleno de los trabajadores, al igual que no cuentan con los implementos de seguridad para protegerse ante los riesgos que se exponen en su área de trabajo, no cuentan con suministro de agua potable durante el recorrido, así como tampoco cuentan con baños ni sala de descanso en el denominado punto de control, aunque cabe destacar que se encontraba en construcción al momento de la inspección.

Al culminar la inspección laboral se evidencio que los trabajadores que prestan un servicio a la empresa contratista no cuentan con seguridad social no tienen conocimiento sobre los planes de formación, los trabajadores perteneciente a la empresa contratista están inscrito en el sistema seguro social, cuentan con planes de formación y se demostró que cuentan con representantes en el comité de seguridad y salud laboral de la empresa.

En cuanto a las condiciones integrales de trabajo que se refieren a condiciones de seguridad, prestaciones sociales, estabilidad laboral, contrato escrito de trabajo y seguridad social, se evidencia una violación a los derechos de estos trabajadores, demostrando que los trabajadores que se encuentran más expuestos son los de los camiones independientes realizando labores que inciden de forma negativa el desenvolvimiento del trabajador $y$ su desarrollo y crecimiento personal.

\section{REFERENCIAS}

Albarracín, J. (2002). La teoría del Riesgo y el Manejo del Concepto Riesgo en las Sociedades Agropecuarias Andinas. En línea

http://bibliotecavirtual.clacso.org.ar/Boli via/cidesumsa/20120903104211/albarra .pdf. Consulta. 2016, Agosto 15

Azuaje, I. (2000).Condiciones de Trabajo. Trabajo de Grado. Trabajo investigación para optar al título de Licenciado en Relaciones Industriales no publicado. Universidad Carabobo. Bárbula

Banchs, M. A. (1990). La propuesta epistemologica de Ignacio Martín-Baró para uma psicologia social na América Latina. Boletin da AVEPSO, 13(3), 12-15

Beck, U., y Zolo, D. (2005). La sociedad global del riesgo. Una conversación entre Ulrich Beck y Danilo Zolo. Sociológica (México), 20(57), 307-327

Constitución de la República Bolivariana de Venezuela (1999). Gaceta Oficial de la República Bolivariana de Venezuela 5.453, Marzo 24,2000.Caracas

Colombet, C., (2013) Nociones de seguridad social. En Delgado de Smith, Yamile y Christian Colombet (coordinadores) Relaciones Industriales reflexiones teóricas y prácticas. Editorial dirección de medios y Publicaciones de la Universidad de Carabobo Venezuela. (p. 91-112) Universidad de Carabobo. Valencia

Creus, A., y Mangosio, J. (2011).Seguridad e Higiene en el Trabajo. Un Enfoque Integral. Editorial Alfa omega. Buenos Aire

Chiavenato, I. (1989). Administración de Recursos Humanos. Editorial Atlas, México Distrito Federal

Chiavenato, I. (2007). Administración de Recursos Humanos. El Capital Humano de las Organizaciones. Mc Graw-Hill. Venezuela

Fernández, R. (2005). Administración de la responsabilidad social corporativa. Thompson Editores. España

Holm, D., y Martin A. (2005) Manual de la sociología del trabajo y las relaciones laborales. Editorial Delta Publicaciones. España

Jodelet, D. (1989). Représentations sociales: Un domaine en expansion. En D. Jodelet (Dir.), Les representations sociales (pp. 31-62). Paris: PUF 
Ley Orgánica del Trabajo, de las Trabajadoras y Los Trabajadores (2012). Gaceta Oficial de la República Bolivariana de Venezuela 6.076, Extraordinaria. Decreto con Rango Valor y Fuerza de Ley Orgánica del Trabajo, de las Trabajadoras y Los Trabajadores. Mayo 07, 2012. Caracas

Ley Orgánica de Prevención, Condiciones y Medio Ambiente de Trabajo (2005). Gaceta Oficial de la República Bolivariana de Venezuela 38.236, Extraordinaria. Decreto con Rango Valor y Fuerza de Ley Orgánica de Prevención, Condiciones y Medio Ambiente de Trabajo. Julio 26, 2005. Caracas

Ley del Seguro Social (2012).Gaceta Oficial de la República Bolivariana de Venezuela 39.912, Extraordinaria. Decreto con Rango Valor y Fuerza de Ley reforma Parcial de la Ley de seguro social. Abril 30, 2005.Caracas

López, M. (1997). La sociología de las relaciones laborales contemporánea. En Holm, Detlev y Martin Antonio (2005). Manual de la sociología del trabajo y las relaciones laborales. (p. 125-177). Editorial Delta Publicaciones. España.

Luhmann, N. (1964).Teorías Actuales de la Sociología de las Relaciones Laborales. En Holm, Detlev y Martin Antonio (2005). Manual de la sociología del trabajo y las relaciones laborales. (p. 182-292). Editorial Delta Publicaciones. España

Luhmann, N., (1986). Fin y racionalidad en los sistemas. Editora Nacional. Madrid.

Neffa, J. (2015). Los Riesgos Psicosociales en el Trabajo Contribución a su Estudio. Libro en línea. Disponible: http://www.ceil-conicet.gov.ar/wpcontent/uploads/2015/11/NeffaRiesgos-psicosociales-trabajo. Consulta: 2016, Marzo, 30

Ordenanza de Reforma a la Ordenanza sobre el régimen del Servicio de Aseo Urbano del Municipio Naguanagua (2008). Gaceta Municipal Nro.960251001 Reforma a la Ordenanza sobre el régimen del Servicio de Aseo Urbano del Municipio Naguanagua. Diciembre 18, 2008. Naguanagua

OIT (2000). Organización Internacional del trabajo Informe sobre el trabajo en el mundo, Oficina Internacional del trabajo, Ginebra, Suiza

OIT (2005) Organización Internacional del trabajo. Informe global de la Declaración de Seguimiento los principios y derechos fundamentales en el trabajo, Ginebra, Suiza

Parra, Y. y Rodríguez, M. (2009). Condiciones y medio ambiente de trabajo de las funcionarias y funcionarios públicos en el marco de la LOPCYMAT y su reglamento parcial. Caso: contraloría municipal de los guayos. Trabajo investigación para optar al título de Licenciado en Relaciones Industriales no publicado, Universidad de Carabobo, Bárbula

Parsons, T. (1951). Teorías Actuales de la Sociología de las Relaciones Laborales. En Holm, Detlev y Martin Antonio (2005). Manual de la sociología del trabajo y las relaciones laborales. (p. 182-292). Editorial Delta Publicaciones. España

Plumise, L. (1991). A Visit with Fred Herzberg. Management Newsletter. En Manzo, Juan (2002). El legado de Frederick, Herzberg. Revista Universidad de EAFIT, (128),

Chile

Porter, L. (1966). Gestión de Personal. Revista Anual de Psicología, (17), 419431

Prigogine, I., y Stengers, I. (1988) Entre el Tiempo y la eternidad. Editorial Fayard.

Reglamento Parcial de la Ley Orgánica de Prevención, Condiciones y Medio Ambiente de Trabajo (2006). Gaceta Oficial de la República Bolivariana de Venezuela 5078, Extraordinaria. Decreto con Rango Valor y Fuerza de Ley

Reglamento Parcial de la Ley Orgánica de Prevención, Condiciones y Medio Ambiente de Trabajo. Diciembre 22, 2006. Caracas 